\title{
Matthias Neugebauer Jesus, Nietzsche und Trump. Wahrheit, Irrtum und Lüge in Religion, Philosophie und Politik
}

\section{Einleitung}

Erkenntnisse im Sinne von wahren Erkenntnissen und Irrtümer im Sinne von nachweislich irrtümlichen oder irreführenden Erkenntnissen gibt und gab es in der Religion und in der an sie anschliessenden Theologie. Irrtümer und Erkenntnisse im beschriebenen Sinne gab und gibt es in Philosophie und den anschliessenden Debatten. Erkenntnisse und Irrtümer sind schliesslich auch typisch für die Politik und den politischen Diskurs. Für die genannten drei Bereiche stehen die Namen Jesu, Nietzsches und Trumps.

Diese Kompilation mag auf den ersten Blick irritieren oder verblüffen. Aber sie besitzt dennoch eine innere Logik. Denn Jesus sagt im Johannesevangelium: «Ich bin der Weg, die Wahrheit und das Leben» (Joh 14,6), eine Einsicht, an die in der anschliessenden Kirchen- und Theologiegeschichte eine ganze Reihe von lebensdienlichen und zum Teil weltverändernden Erkenntnissen generiert wurden. Wichtig an dieser Stelle ist zunächst nur, dass es sich um eine strikt religiöse Wahrheit handelt, also eine Wahrheit, die unabhängig von menschlichen Geltungsansprüchen und allein im Modus des religiösen Glaubens subsistiert.

Nietzsche hat seinen Platz in dieser Reihe, weil er die Möglichkeit suffizienter, sprich wahrer Erkenntnis im Ganzen bezweifelt hat. Seine Einsichten gipfeln in dem Spitzensatz: «Wahrheit ist die Art von Irrtum, ohne welche eine bestimmte Art von lebendigen 
Wesen nicht leben könnte». ${ }^{1}$ Dahinter steht Nietzsches These, dass es Wahrheit nicht gibt, sondern dass sich alles als Wahrheit Beschreibbares, wenn überhaupt, ereignet. Und solche Wahrheitsmomente ereignen sich, wenn sich das, was Nietzsche als den «Übermenschen» diviniert, performiert: Was also der «Übermensch» sagt, ist Wahrheit. Der «Übermensch» irrt nicht. Seine Erkenntnisse sind wahr.

Und der amtierende Präsident Donald Trump wird aufgerufen, nicht weil es in der Politik immer schon einen eigenen Umgang mit Erkenntnissen und Irrtümern gegeben hat, sondern weil dieser US-Präsident in bisher nie dagewesener Weise mit Irrtum und Wahrheit umgeht. Laut der Washington Post hat Präsident Trump in seiner bisherigen Amtszeit jeden Tag etwa 8,3 falsche oder irreführende Behauptungen geäussert. Direkt vor den Zwischenwahlen, den Mid Term Elections im Herbst 2018, stieg diese Zahl auf bis zu 30 an. ${ }^{2}$ Die Washington Post führt in dieser Hinsicht die sogenannte «Fact Checker's ongoing database of the false or misleading claims made by President Trump since assuming office». ${ }^{3}$ Und am 30. Januar 2019 war dort zu lesen: «In 730 days, President Trump has made 8158 false or misleading claims». ${ }^{4}$

Wahrheit, Irrtum und Lüge in Religion, Philosophie und Politik - das soll im Folgenden genauer angeschaut werden, und wir zäumen das Ross gewissermassen von hinten auf und beginnen mit Donald J. Trump, dem 45. Präsidenten der Vereinigten Staaten von Amerika.

Friedrich Nietzsche: Nachgelassene Fragmente (April-Juni 1885) 34 [253], in: Sämtliche Werke. Kritische Studienausgabe [= KSA], hg. von Giorgio Colli und Mazzino Montinari, München, Berlin, New York 1980, XI 506 (Hervorhebung M.N.). http:// www.spiegel.de/politik/ausland/donald-trump-vor-denmidterm-wahlen-luegenrekord-gebrochen-a-1236589.html (30.1.2019).

3 https://www.washingtonpost.com/graphics/politics/trump-claimsdatabase (30.1.2019).

4 Ebd. 
147 Matthias Neugebauer: Wahrheit, Irrtum und Lüge

Irrtum und Erkenntnis in der Politik

Donald Trump - «Alternative Wahrheiten» und «Fake News»

Dass in der Politik ein zum Teil fragwürdiges Verhältnis zu wahren Erkenntnissen und zu Irrtümern subsistiert, ist keineswegs neu und mitnichten eine Signatur der Moderne. Natürlich gibt es immer rühmliche Ausnahmen. Es sei hier als gewissermassen leuchtendes Beispiel der Heerführer und Staatsmann der Antike Marcus Aurelius (121-180 n. Chr.) aufgerufen, der seiner Wahrheitsliebe wegen schon in jungen Jahren «verissimus» genannt wurde. Er schreibt in seinen berühmten Selbstbetrachtungen: «Kann mir jemand überzeugend dartun, dass ich nicht richtig urteile oder verfahre, so will ich's mit Freuden anders machen. Suche ich ja nur die Wahrheit, sie, von der niemand je Schaden erlitten hat. Wohl aber erleidet derjenige Schaden, der auf seinem Irrtum und auf seiner Unwissenheit beharrt». ${ }^{5}$

Aber das ist eher die Ausnahme denn die Regel. Und es darf bezweifelt werden, dass Marcus Aurelius diesem Anspruch immer gerecht geworden ist. Denn Politik ist über Weiten ein schmutziges Geschäft. Dass um eines politischen Vorteils willen, um Macht zu erlangen oder zu erhalten oder um Interessen durchzusetzen, irreführende Behauptungen in die Welt gesetzt werden, ist die traurige Realität, und diese hat auch eine lange Geschichte. Erinnert sei beispielsweise das alte Ägypten und an den wohl bedeutendsten Pharao des Neuen Reiches, Ramses II. (reg. 1292-1225 v. Chr.). Er zog im Jahr 1287 v. Chr. gegen die konkurrierenden Hethiter in die Schlacht, wo es bei Kadesch am Orontes fast zu einer Katastrophe kam. Quasi in letzter Minute konnte sich Ramses' Truppen einem Umfassungsmanöver durch die Hethiter entziehen. Ramses hat dann (wieder zurück im Reich) den Ausgang der Schlacht in einen gewaltigen Sieg umdichten lassen. ${ }^{6}$ Dort ist dann zu lesen, wie ein

5 Marc Aurel: Selbstbetrachtungen, übers. u. hg. von Albert von Wittstock, Stuttgart 1986, VI 21.

6 Vgl. Egon Friedell: Kulturgeschichte Ägyptens und des Alten Orients, München ${ }^{61992, ~ 310-314, ~ h i e r ~ 311: ~ « D i e ~ a ̈ g y p t i s c h e n ~ H o f h i s t o r i o g r a p h e n ~}$ 
strahlender Sieger Ramses II. die Hethiter in den Orontes wirft, was so keinesfalls passiert ist.

Dieses Muster lässt sich durch die gesamte Geschichte hindurch beobachten. Es hält sich durch bis zur sogenannten десинформация in der russischen Zaren- und Sowjet-Zeit und findet Ausdruck in dem oft auf den US-Senator Hiram Johnson (1866-1945) zurückgeführten Satz: «Das erste Opfer des Krieges ist die Wahrheit». Ein trauriger Höhepunkt wird erreicht mit der Propaganda des Nationalsozialismus, die für derartige Irreführungen ein eigenes Reichsministerium einrichteten, das «Reichsministerium für Volksaufklärung und Propaganda» unter Josef Goebbels. ${ }^{7}$ Allerdings: Einmal von Nordkorea abgesehen, sind wir heutigen Tags auf dieser Welt von derartigen Irreführungs-Exzessen weit entfernt, vor allem in der westlichen Welt und damit auch den USA.

Aber: Irreführungen sind weiter an der Tagesordnung. Alle US-Präsidenten haben gelogen und irreführende Behauptungen in die Welt gesetzt, worauf beispielsweise Bill Press in der Chicago Press vom 19. Oktober 2017 hingewiesen hat: «Of course, Trump's not the first president not to tell the truth. President Eisenhower covered up the mission of U-2 spy pilot Francis Gary Powers. Ronald Reagan lied about selling arms to the Contras. Bill Clinton lied about Monica Lewinsky». ${ }^{8}$ Aber es ist unübersehbar, dass der amtierende US-Präsident, Donald J. Trump, alles in den USA bisher Dagewesene in den Schatten stellt: die Infragestellung des Klimawandels, die willkürliche Diffamierungen von Einzelpersonen und der Presse, unbekümmertes Äussern von diametral entgegengesetzten Aussagen seien hier nur als Spitze des sprichwörtlichen Eisbergs aufgerufen.

haben die Schlacht von Kadesch später in einen großen Sieg umgedichtet, während sie bloß ein bravouröser Rückzug war.»

7 Das Reichsministerium für Volksaufklärung und Propaganda wurde am 13.3.1933 eingerichtet und Josef Goebbels Reichspropagandaminister; vgl. William Lawrence Shirer: Aufstieg und Fall des Dritten Reiches, Bindlach 1990, 193.

8 Bill Press: Does Donald trump ever tell the truth?, in: Chicago Press, 19.10.2017. 
In dieser Beziehung wurde und wird vor allem in den USA seit seinem Amtsantritt eine leidenschaftliche Debatte darüber geführt, was sich hinter diesem zum Teil erratischen Verhalten verbirgt. Eine Schlüsselrolle spielt an dieser Stelle zunächst der Ausdruck der und die Diskussion um die sogenannten «alternativen Fakten» («alternative facts»).

Man kann den Auftakt der diesbezüglichen Debatte sehr klar orten. Es war am 20. Januar 2017, als Trump-Beraterin Kallyane Conway in einem Interview mit Chuck Todd in der Sendung «Meet the Press» Stellung dazu nahm, ob bei Obamas oder Trumps Amtseinführung mehr Menschen auf der sogenannten National Mall, also der Achse zwischen dem Capitol und dem Lincoln Memorial, gewesen seien. Der damalige Pressesprecher Sean Spicer hatte kurz zuvor behauptet, noch nie seien mehr Menschen anlässlich der Inauguration eines amerikanischen Staatsoberhauptes auf der National Mall gewesen, was schnell im Netz und in anderen Medien als Unfug beziehungsweise Unwahrheit - oder wenn man es noch wohlwollend einschätzen würde - wenigstens als Irrtum aufgedeckt werden konnte. Conway liess sich indes nicht beirren und stärkte Spicer den Rücken eben mit der Formulierung, es gäbe eben so etwas wie «alternative Fakten»: «Don't be that overly dramatic about it, Chuck. You're saying it's a falsehood, and they're giving - Sean Spicer, our press secretary, gave alternative facts to that». ${ }^{9}$ Auf die Rückfrage, ob denn sogenannte «alternative Fakten» nicht einfach als «Lügen» zu qualifizieren seien («Alternative facts are not facts. They are falsehood»), ${ }^{10}$ konterte Conway, es gäbe keine gesicherte Methode, Menschenmengen auf der National Mall zu bestimmen, und überhaupt sei ihr Gesprächspartner kein Informator, sondern nur Kommentator von Fakten. Trump sekundierte seinen Mitarbeitern Conway und Spicer, indem er alle Informationen, die der Darstellung von Spicer und Conway zuwiderliefen, als «fake news»

9 https://www.nbcnews.com/meet-the-press/meet-press-01-22-17n710491 (30.1.2019 [Hervorhebung M.N.]).

Ebd. 
bezeichnete, eine Vokabel, die Trump seitdem als Präsident unentwegt bemüht.

Es sei an dieser Stelle dahingestellt, ob sich hier bereits die von Donald Trump in seinem Buch The Art of Deal von 1987 und dem Theorem der «wahrheitsgemässen Übertreibung» («truthful hyperbole») Durchbruch verschafft, oder ob das Problem tiefer liegt. Nicht zu übersehen ist indes, dass mit den «alternative facts» und den ihnen korrespondierenden «fake news» ein politischer Stil in den öffentlichen Diskurs Einzug gehalten hat, der in dieser Form in den USA bislang unbekannt war. Und dieses Konzept scheint sich zu verfestigen, nicht nur durch Trumps unablässiges Anathematisieren von Informationen als «fake news», sondern auch durch seine Mitarbeiter. Vor dem Hintergrund der sogenannten Russland-Affäre und der Arbeit des Sonderermittlers Robert Mueller sprach der ehemalige New-Yorker Bürgermeister und jetzige Trump-Anwalt Rudy Giuliani jüngst in einem Interview (wieder in «Meet the Press» und wieder mit Chuck Todd) am 19. August 2018 von «Versionen der Wahrheit» («somebody's version of the truth»). ${ }^{11}$ Und auf den Einwurf von Chuck Todd, die Wahrheit sei doch die Wahrheit, antwortete Giuliani dann mit dem berühmt berüchtigt gewordenen Satz «Nein, es ist nicht die Wahrheit [...] Die Wahrheit ist nicht die Wahrheit» («No it isn't truth [...] truth isn't truth»). ${ }^{12}$

Da Trump mittlerweile eine derart grosse Anzahl an Lügen und irreführenden Äusserungen in die Welt gesetzt hat, wurde das «Lügen-Phänomen Trump» beziehungsweise der «Lügner Trump» bereits zum Gegenstand systematischer Betrachtungen. Linda Qiu hat in dieser Beziehung am 29. Dezember 2018 in der New York Times ein Viererschema ausgemacht, das die häufigsten Lügenmuster Trumps aufzeigt. Das sind im Einzelnen: die gleichsam endlose Wiederholung und aufblasende Übertreibung von

\footnotetext{
11 https:/ / www.nbcnews.com/meet-the-press/meet-press-august-192018-n901986 (30.1.2019).

12 https://www.nbcnews.com/meet-the-press/meet-press-august-192018-n901986 (30.1.2019).
} 
vermeintlich wahren Erkenntnissen («repetition and inflation»), Verschiebestrategien und Ablenkungsmanöver («shifting and deflecting»), bewusst irreführende Ungenauigkeit und PhantasieDetails («misleading vagueness and fanciful details») und die Erfindung von Strohmännern («inventing straw men»). ${ }^{13}$

Ergänzend kann an dieser Stelle hinzugefügt werden, dass Trump zusätzlich das Verschwörungs-Narrativ vom «Heimlichen Staat» («Deep State») geschickt mitbewirtschaftet. Dieses Narrativ hat der Politikwissenschaftler Bernd Schlipphak in einem Interview im Deutschlandfunk am 10. November 2018 folgendermassen beschrieben: «In den USA [gibt es] die Vorstellung eines sogenannten 〈Deep-State〉. Das ist die Vorstellung von einer geheimen Regierungselite, die irgendwo in Washington sitzt, in geheimen Hinterzimmern und alles ausklüngelt, was [...] politisch läuft. Und die grosse Hoffnung von Teilen von Trumps Wählerschaft ist, dass Trump der erste Outsider ist, der jetzt nach Washington geht und mit diesem «Deep-State〉 aufräumt, sozusagen die Verschwörung durchbricht.» ${ }^{14}$ Natürlich gibt es diesen «Heimlichen Staat» («Deep State») nicht, aber zusammengenommen mit den vorher genannten Punkten ergibt das im Ganzen einen höchst undurchschaubaren Cocktail der Unwahrheit.

In der Bewertung dieses politischen Gestus haben sich vor allem drei Erklärungshinsichten etabliert. Die erste Erklärungshinsicht kam relativ schnell auf und bescheinigte Trump eine kognitive Beeinträchtigung, die womöglich aus Altersstarrsinn oder Demenz resultiert. Der amerikanische Journalist Bill Press hat dies in der Chicago Press folgendermassen beschrieben: «Donald Trump's the first president to lie about everything. Indeed, he lies with such facility you wonder if he's so divorced from reality that he doesn't even know he's lying. He's clearly unable to tell the difference,

13 https://www.nytimes.com/2018/12/29/us/politics/trump-factcheck.html?action $=$ click \&module $=$ Top $\% 20$ Stories\&pgtype $=$ Homepage (30.1.2019).

14 https:// srv.deutschlandradio.de/ dlf-audiothek-audioteilen.3265.de.html?mdm:audio_id=689856\# (30.1.2019). 
or unwilling to take the time to learn, between what is true and what he wants to be true.» ${ }^{15}$

Trump selbst war an dieser Stelle bemüht, jeden Anschein von kognitiver Beeinträchtigung oder Demenz zu zerstreuen und absolvierte proaktiv den sogenannten MoCA-Test (Montreal Cognitive Assessment). Der Test prüft Erinnerung, Sprache, Orientierung, Steuerungsfähigkeit und Aufmerksamkeit. Trump erreichte die maximale Punktzahl von 30 Punkten. Und es wurde betont, dass ab 26 Punkten die Ergebnisse normal seien. Die Aussagekraft des Testes ist allerdings umstritten und dann Gegenstand einer eigenen Neben-Debatte geworden.

Der zweite Erklärungsversuch geht dahin, dass Trumps politischer Stil der eines gerissenen Taktikers ist. Demnach kennt Trump den Unterschied zwischen wahren Erkenntnissen und irreführenden Behauptungen sehr wohl. Aber wenn es darum geht, seine politische Agenda durchzusetzen, schert er sich nicht um diesen Unterschied, sondern setzt ihn ganz bewusst ausser Kraft. Aufmerksamkeit in dieser Hinsicht erregt hat eine Äusserung Trumps in einem Interview mit dem Sender ABC-News vom 31. Oktober 2018. Dort wurde Trump darauf angesprochen, er habe doch dem amerikanischen Volk vor Amtsantritt versprochen immer die Wahrheit zu sagen und Trump antwortete: «Well, I try. I do try [...] and I always want to tell the truth [...]. When I can, I tell the truth». ${ }^{16}$

«When I can, I tell the truth». Die amerikanische Schriftstellerin Irene Dische hat dies (in einem Interview mit Michael Köhler im Deutschlandfunk vom 11. November 2018) folgendermassen übersetzt: «Ich sage die Wahrheit, wenn es mir passt.» ${ }^{17}$ Und sie führt dieses Verhalten von Trump zusätzlich darauf zurück, dass er sich

B. Press: Does Donald trump ever tell the truth? (Hervorhebungen M.N.).

16 https://www.washingtonpost.com/politics/when-i-can-i-tell-thetruth-trump-pushes-back-against-his-peddling-offalsehoods / 2018/11/01/e8278d68-ddbe-11e8-85df7a6b4d25cfbb_story.html (30.1.2019 [Hervorhebung M.N.]).

17 https://www.deutschlandfunk.de/trump-und-die-medien-wir-habenin-amerika-eine-art.694.de.html?dram:article_id=432933 (30.1.2019). 
permanent im (Wahl-)Kampf-Modus befände und für ihn entsprechend gelte: «All fair in love and war» - «Im Krieg und in der Liebe ist alles erlaubt». ${ }^{18}$

Ein drittes Erklärungsmuster hebt darauf ab, dass Trump derart von sich überzeugt sei, dass er glaube, er generiere per se nur wahre Erkenntnisse, beziehungsweise alles, was er verlautbaren lasse, sei, weil er es verlautbaren lasse, die Wahrheit und nichts als die Wahrheit. In dieser Beziehung hat ein Tweet Trumps für nachhaltige Aufmerksamkeit gesorgt, in dem er sich ohne jeden Anflug von Selbstzweifel als Genie erklärt: «I went from VERY successful businessman, to top T.V. Star to President of the United States (on my first try). I think that would qualify as not smart, but genius ... and a very stable genius at that!» ${ }^{19}$

Es war wieder Rudy Giuliani, der Trumps Ego in dieser Hinsicht befeuerte. Im Kontext der sogenannten Russlandaffäre und der Frage, ob Präsident Trump das Rechtssystem behindere, äusserte Giuliani, dass Trump «von Amts wegen das Recht nicht behindern [könne], da er das Recht sei». ${ }^{20}$ Das deutsche Nachrichtenmagazin Der Spiegel bezeichnete dies prompt als eine «historisch beispiellose und juristisch umstrittene Anmaßung». ${ }^{21}$ Und nicht nur das. In einer Linie damit steht die fast surreale Szene, in der der (aus dem türkischen Hausarrest zurückgekehrte) Pastor Andrew Brunson vor Trump kniet und für ihn um «übernatürliche Weisheit» («supernatural wisdom») betet. ${ }^{22}$ Dazu passt schliesslich,

18 Ebd.

19 Tweet von @realDonaldTrump vom 6.1.2018 (Hervorhebung M.N.).

20 http:/ / www.spiegel.de/politik/ausland/donald-trump-derallmaechtige-praesident-a-1210978.html (30.1.2019).

21 Ebd.

22 Der Wortlaut des Gebetes lautet im Englischen: «Father God I ask that you'd pour out your Holy Spirit on President Trump, that you give him supernatural wisdom to accomplish all the plans you have for this country. I ask that you give him wisdom to lead this country into righteousness. I ask that you'd give him perseverance and endurance and courage to stand for truth. I ask that you protect him from slander from enemies, from those who would undermine. I ask that you'd make him a great blessing to this country and fill him with your wisdom and strength and 
was Trump laut der Süddeutschen Zeitung vom 11. August 2018 in einer Rede zu US-Kriegsveteranen in Kansas City sagte: «Merkt euch: Was ihr seht und lest, passiert nicht wirklich. [...] Glaubt einfach uns.» ${ }^{23}$

Trump erinnert in gewisser Weise ganz von selbst an Nietzsche. ${ }^{24}$ Denn scheinbar teilt er nicht nur Nietzsches entschiedene Reserve «gegen die Weichlichkeit der demokratischen Ideen», ${ }^{25}$ sondern vor allem das quasi unerschütterliche Überzeugtsein von der eigenen Genialität - in Verbindung mit dem Anspruch, immer richtig zu liegen - erinnert an die von Nietzsche divinierte Gestalt des Übermenschen, der es sich deshalb im Folgenden zuzuwenden gilt.

Irrtum und Erkenntnis in der Philosophie

Friedrich Nietzsche - «Wahrheit [...] ist Irrthum ...»

Nietzsche gilt in der Philosophiegeschichte unter anderem als der «Alleszertrümmerer». Diese Titulierung rührt daher, dass er dem letzten von ihm selbst veröffentlichen Buch Götzen-Dämmerung den alternativen Titel gab: «oder Wie man mit dem Hammer philosophirt». ${ }^{26}$ Der «Epilog», der einen Auszug aus Also sprach Zarathustra wiedergibt, ist überschrieben mit «Der Hammer redet». ${ }^{27}$ Und im

perseverance. And we bless him. Make him be a great blessing to our country. In Jesus' name, we bless you. Amen»,

https://www.christianheadlines.com/contributors/michael-

foust/pastor-brunson-prays-with-trump-give-wisdom-lead-country.html (30.1.2019).

23 https://www.sueddeutsche.de/politik/us-politik-wie-trump-versuchtdie-realitaet-zu-besiegen-1.4086908 (30.1.2019).

Der Link von Trump zu Nietzsche ist auch bereits in der amerikanischen Presse aufgetaucht: Steven Poole: «Truth isn't truth»: so, is that true?, in: The Guardian, 13.8.2018, https:// www.theguardian.com/books/2018/aug/23/truth-isnt-truthso-should-we-expect-lies-from-donald-trump (30.1.2019). F. Nietzsche: Nachlass, April-Juni 188534 [203], in: KSA XI 489.

26 F. Nietzsche: Götzen-Dämmerung, in: KSA VI 55.

27 Ebd., 161. 
«Grablied» aus dem zweiten Teil des Zarathustra singt Zarathustra am Ende: «Ja, noch bist du mir aller Gräber Zertrümmerer: Heil dir, mein Wille». ${ }^{28}$

Nietzsches Hammer arbeitet sich an den grossen Themen der Philosophie- und Theologiegeschichte ab: Metaphysik, Theologie, Moral, Geist, Vernunft, Denken, Bewusstsein, Seele usw. ${ }^{29}$ Und zu den Dingen, die in seinem Denken unter den Hammer kommen, gehören zweifellos auch Erkenntnis und Wahrheit, mit den Worten Nietzsches «der Trieb zur Wahrheit, zum reinen Erkennen». ${ }^{30}$ Und in Bezug auf Wahrheit und Erkenntnis kommt Nietzsche zu der Einsicht, dass das Konzept Wahrheitserkenntnis als Ganzes zu verabschieden sei. Er hält im späten Nachlass Mitte der 1880er Jahre dezidiert fest, dass «Erkenntniß Fälschung des Vielartigen und Unzählbaren zum Gleichen, Ähnlichen, Abzählbaren ${ }^{31}$ ist. Weiter heisst es: «Denken ist fälschliches Umgestalten». ${ }^{32}$ Und er notiert: «Es giebt vielerlei Augen. Auch die Sphinx hat Augen: und folglich giebt es vielerlei 〈Wahrheiten〉, und folglich giebt es keine Wahrheit». ${ }^{33}$

Um nicht den Eindruck zu erwecken, es werde im Folgenden ein unkontrolliertes Zitaten-Potpourri abgewickelt, wird grosso modo chronologisch vorgegangen; wohl wissend, dass Nietzsches Werk selbst zu grossen Teilen ein Potpourri von ganz unterschiedlichen

F. Nietzsche: Also sprach Zarathustra. Ein Buch für Alle und Keinen, in: KSA IV 9-408, 145. Vgl. dazu auch das «Zertrümmern und Verhöhnen der alten Begriffsschranken»; F. Nietzsche: Ueber Wahrheit und Lüge im außermoralischen Sinne, in: KSA I 873-890, 889.

Vgl. dazu: Thomas Vašek: Seele eine unsterbliche Idee. Warum wir mehr sind als die Summe unserer Teile, Kiel 2010, 285: «Nietzsche will alles zertrümmern, was die Philosophen über die Jahrhunderte aufgebaut haben. Es gibt weder Geist, noch Vernunft, noch Denken, noch Bewusstsein, noch Seele, noch Wille, noch Wahrheit: alles Fiktionen, die unbrauchbar sind.» F. Nietzsche: Ueber Wahrheit und Lüge, 882.

31 F. Nietzsche: Nachlass, April-Juni 188534 [252], in: KSA XI 506 (Hervorhebung im Original, dort gesperrt).

32 Ebd.

33 F. Nietzsche: Nachlass, April-Juni 188534 [223], in: KSA XI 498. 
und atemporalen Aphorismen sowie diskursiven Texten darstellt. Nietzsches Kritik am Systemgedanken steht freilich immer auf Kriegsfuss mit allen (auch chronologischen) Systematisierungsversuchen. Deshalb darf, soll und muss Nietzsche auch immer diachron gelesen werden. Er hätte das so gewollt: «Ein Aphorismus, rechtschaffen geprägt und ausgegossen, ist damit, dass er abgelesen ist, noch nicht entziffert; vielmehr hat nun erst dessen Auslegung zu beginnen, zu der es einer Kunst der Auslegung bedarf. [...] Freilich thut, um dergestalt das Lesen als Kunst zu üben, Eins vor Allem noth, was heutzutage gerade am Besten verlernt worden ist - und darum hat es noch Zeit bis zur Lesbarkeit meiner Schriften -, zu dem man beinahe Kuh und jedenfalls nicht moderner Mensch sein muss: das Wiederkäuen». ${ }^{34}$

Das Wahrheits-Thema hat Nietzsche schon in seiner Frühzeit umgetrieben. 1873 verfasste er die (zu seinen Lebzeiten unveröffentlicht gebliebene) Schrift Ueber Wahrheit und Lüge im aussermoralischen Sinne. Bereits hier gilt ihm die Arbeit des wahrheitssuchenden menschlichen Intellekts folgendermassen: «[H]ier ist die Täuschung, das Schmeicheln, Lügen und Trügen, das Hinter-dem-RückenReden, das Repräsentieren, das im erborgten Glanze Leben, das Maskiertsein, die verhüllende Konvention, [...] daß fast nichts unbegreiflicher ist, als wie unter den Menschen ein ehrlicher und reiner Trieb zur Wahrheit aufkommen konnte. Sie sind tief eingetaucht in Illusionen und Traumbilder». ${ }^{35}$ Nietzsche bezeichnet Wahrheitsansprüche im Folgenden als Metaphern und antwortet auf die Frage «Was ist Wahrheit?» mit dem berühmten Satz: «Ein bewegliches Heer von Metaphern, Metonymien, Anthropomorphismen, kurz eine Summe von menschlichen Relationen, die, poetisch und rhetorisch gesteigert, übertragen, geschmückt wurden, und 245-412, Vorrede, 8 (Hervorhebung M.N.). Zum Motiv des Wiederkäuens, das - nebenbei bemerkt - an Luthers berühmte Auslegung des ersten Psalms erinnert vgl. auch F. Nietzsche: Also sprach Zarathustra, 334.

35 F. Nietzsche: Ueber Wahrheit und Lüge, 876. 
die nach langem Gebrauche einem Volke fest, canonisch und verbindlich dünken: die Wahrheiten sind Illusionen, von denen man vergessen hat, dass sie welche sind.» ${ }^{36}$

Diese Einsicht vom Irrtumscharakter der Wahrheit ist eingebettet in die These von der «Irrthümlichkeit der Welt», ${ }^{37}$ wie sie Nietzsche dann in seiner mittleren Phase unter anderem in Jenseits von Gut und Böse entfaltet hat. Der Sache nach tauchte dieser Vorstellungskreis bereits auch in Nietzsches Erstschrift, in der Geburt der Tragödie, auf und zwar als der sogenannte Apollonische Schein. ${ }^{38}$ War aber die Apollonische Umschleierung in der Geburtsschrift noch erlösende Gegenvision in Konfrontation mit dem Ur-Einen, so erscheint sie in Jenseits von Gut und Böse als konstitutives Moment der Konstruktion von Wirklichkeit in Gestalt von Metaphern und Interpretationen. Hinter der notwendig scheinhaft konzeptualisierten Wirklichkeit lässt sich nichts Erscheinendes, kein «Wesen der Dinge» ${ }^{39}$ postulieren. Welt ohne Schein gibt es im strengen Sinne nicht; genauso wenig wie harte Tatsachen. ${ }^{40}$ Die Wirklichkeit ist fiktional und zwar notwendig

Ebd., 880.

F. Nietzsche: Jenseits von Gut und Böse. Vorspiel einer Philosophie der Zukunft, in: KSA V 9-243, [34], 53 (im Original zum Teil gesperrt).

Vgl. dazu Matthias Neugebauer: Konzepte des «Bios»: Leben im Spannungsfeld von Organismus, Metaphysik, Molekularbiologie und Theologie, Göttingen 2009, 103-114.

F. Nietzsche: Ueber Wahrheit und Lüge, 879.

Vgl. dazu auch die Nachlassnotiz von 1886: «Thatsachen gibt es nicht, nur Interpretationen»; F. Nietzsche: Nachlass, Ende 1886-Frühjahr 18877 [60], in: KSA XII 315. Vgl. dazu auch die These aus der Fröhlichen Wissenschaft: «Der Gesammt-Charakter der Welt ist dagegen in alle Ewigkeit Chaos, nicht im Sinne der fehlenden Nothwendigkeit, sondern der fehlenden Ordnung, Gliederung, Form, Schönheit, Weisheit, und wie alle unsere ästhetischen Menschlichkeiten heissen»; F. Nietzsche: Die fröhliche Wissenschaft, in: KSA III 468. Zum Theorem des Irrtums beziehungsweise Scheins vgl. auch ausführlicher Volker Gerhardt: Vom Willen zur Macht. Anthropologie und Metaphysik am exemplarischen Fall Friedrich Nietzsches, Berlin New York 1996, 231; Alexander Hogh: Nietzsches Lebensbegriff. Versuch einer Rekonstruktion, Stuttgart, Weimar 2000, 56-58; Robert Rethy: «Schein» in Nietzsche's Philosophy, in: Keith 
fiktional. Dies hält Nietzsche jedoch für unproblematisch. Lapidar fragt er: «Warum dürfte die Welt, die uns etwas angeht, nicht eine Fiktion sein?» ${ }^{41}$

Dieses denkerische Integral von Fiktion und Realität nennt Nietzsche eine Station später «Wille zur Macht». Der Wille zur Macht wird vor allem in Also sprach Zarathustra entfaltet und erscheint zunächst als die formale Struktur der Selbstüberwindung. ${ }^{42}$ Dies meint des Näheren ein Aus-sich-selbst-sich-selbst-Überwinden. ${ }^{43}$ Der Sache nach tauchte das Motiv der Selbstüberwindung bereits auf. Die beschriebene Genese eines freien Geistes, wie sie im Kontext von Menschliches, Allzumenschliches begegnete, ist faktisch nichts anderes als die im Modus von Loslösung und Selbstergreifung beschriebene Antizipation der im Zarathustra begegnenden Struktur der Selbstüberwindung. ${ }^{44}$ Diese expliziert Nietzsche im Abschnitt «Von der Selbst-Ueberwindung» anhand der Dialektik von Gehorchen und Befehlen. Es ist nämlich dem Gelingen von Selbstüberwinden charakteristisch, «dass es gehorcht und befiehlt und befehlend noch Gehorsam übt». ${ }^{45}$ Der Wille zur Macht ist also gekennzeichnet durch Selbstüberwindung, ${ }^{46}$ und dazu gehören

Ansell-Pearson: Nietzsche and modern German Thought, London 1991, 59-67; Claus Zittel: Irrtum und Schein, in: Henning Ottmann (Hg.): Nietzsche-Handbuch. Leben - Werk - Wirkung, Weimar 2000, 257-259, hier 258.

41 F. Nietzsche: Jenseits von Gut und Böse, 54.

42 Selbstüberwindung darf dabei keineswegs mit der bei Nietzsche auch prominenten Figur der Selbstauflösung verwechselt werden (vgl. Claus Zittel: Selbstauflösungsfiguren bei Nietzsche, Würzburg 1995, 10), auch wenn Selbstüberwindung partiell auch Selbstauflösung beinhaltet (vgl. Ingo Christians: Selbst, in: H. Ottmann: Nietzsche-Handbuch, 321-324, hier 324). Zur Selbstüberwindung vgl. auch Lester H. Hunt: Origin of Virtue, London, New York 1991, 70-71; Charles E. Scott: The Question of Ethics. Nietzsche, Foucault Heidegger, Indianapolis 1990, 15-45.

43 Vgl. F. Nietzsche: Also sprach Zarathustra, 149.

44 Vgl. dazu M. Neugebauer: Konzepte des «Bios», 169-175.

$45 \quad$ F. Nietzsche: Also sprach Zarathustra, 147.

46 «[...], dass das Leben sich immer wieder selber überwinden muss!»; ebd., 130. 
Kampf, ${ }^{47}$ Streit, ${ }^{48}$ Wachstum und Steigerung ${ }^{49}$ sowie immer auch Schöpferisches und Schaffendes, ${ }^{50}$ alles mit dem Ziel, die eigene Selbstmächtigkeit zu steigern. Die Welt und das Weltgeschehen sind zum Schluss nichts anderes als ein Kampf von Willens- und Machtvektoren.

Der interpretative Zugriff auf die Welt vollzieht sich also primär im selbstmächtigen Setzen der massgeblichen Metaphern in Bezug von Wahrheitserkenntnis, Geschichte und Werten, was Nietzsche auch als «Perspektivismus»" ${ }^{51}$ beschreiben kann. Nietzsche spricht in diesem Zusammenhang auch von der «Perspektiven-Optik des Lebens».52 Das meint, dass der interpretative Zugriff auf die Welt im Idealfall allein aus der Perspektive des selbstmächtigen Individuums erfolgt. Die Welt als Gefüge von Machtvektoren erweist sich so als unendlich perspektivisch, wobei die perspektivischen Machtvektoren freilich wiederum graduell abgestuft sind: Herren und Sklaven, Übermensch und Herde.

Der Übermensch als der Mensch, der sich (zum Übermenschen) überwunden hat, performiert sich nicht willkürlich. Einerseits steht der Übermensch für die Realisierung bestimmter Kardinaltugenden, die die Trias von Ehrlichkeit, Tüchtigkeit und vor allem Wahrhaftigkeit umfasst. ${ }^{53}$ Und andererseits (und an dieser Stelle

«Ist in allem Leben nicht selbst - Rauben und Totschlagen?»; ebd., 253; «Und indem ihr tötet, seht zu, dass ihr das Leben rechtfertiget!»; ebd., 45. «Aber alles Leben ist Streit um Geschmack und Schmecken!»; ebd., 151. «Steigen will das Leben und steigend sich überwinden»; ebd., 130. «Wollen befreit: denn Wollen ist Schaffen: so lehre ich. Und nur zum Schaffen sollt ihr lernen»; F. Nietzsche: Also sprach Zarathustra, 258 (im Original teilweise gesperrt]). Vgl. auch den «Schaffens-Willen», in: ebd., 111.

1 F. Nietzsche: Nachlass, Ende 1886-Frühjahr 18877 [60], in: KSA XII 315. Zum Begriff der Perspektive bei Nietzsche vgl. Claus Zittel: Perspektivismus, in: H. Ottmann: Nietzsche-Handbuch, 299-301, hier 300. Der Sache nach finden sich bereits in Menschliches, Allzumenschliches erste Ausführungen zur Perspektivität.

F. Nietzsche: Jenseits von Gut und Böse, 26.

Vgl. F. Nietzsche: Unzeitgemässe Betrachtungen. Zweites Stück: Vom Nutzen und Nachteil der Historie für das Leben, in: KSA I (10.), 333-334. 
scheint gewissermassen ein theologisches Motiv auf, das für die Spätphilosophie prägend ist): Der Übermensch performiert sich wenn man so will - sub specie aeternitatis, also unter dem Gesichtspunkt der Ewigkeit. Die Performation des Willens zur Macht ist nun gebunden an Nietzsches Lehre von der ewigen Wiederkunft des Gleichen.

Der Gedanke der ewigen Wiederkunft ist eines der grossen, wenn nicht das Thema im Zarathustra. Nietzsche notiert rückblickend: «Die Grundconception des Werks [sc. Also sprach Zarathustra], der Ewige-Wiederkunfts-Gedanke, diese höchste Formel der Bejahung, die überhaupt erreicht werden kann -, gehört in den August des Jahres 1881: er ist auf ein Blatt hingeworfen mit der Unterschrift: «6000 Fuss jenseits von Mensch und Zeit». Ich gieng an jenem Tage am See von Silvaplana durch die Wälder; bei einem mächtigen pyramidal aufgethürmten Block unweit Surlei machte ich Halt. Da kam mir dieser Gedanke.» ${ }^{54}$

Mit dem fraglichen Gedanken ging Nietzsche freilich schon etwas früher schwanger. Er taucht kurz vor dem Zarathustra auf am Ende der Fröhlichen Wissenschaft, und er wird dabei explizit als das «grösste Schwergewicht» ${ }^{55}$ eingeführt. Und dieses Schwergewicht erscheint sekundierend im Aphorismus 341 der Fröhlichen Wissenschaft als hypothetischer Ratschlag eines nächtlichen Dämons. Und der Dämon prophezeit: «Dieses Leben, wie du es jetzt lebst und gelebt hast, wirst du noch einmal und noch unzählige Male leben müssen; und es wird nichts Neues daran sein, sondern jeder Schmerz und jede Lust und jeder Gedanke und Seufzer und alles unsäglich Kleine und Grosse deines Lebens muss dir wiederkommen, und Alles in der selben Reihe und Folge [...]. Die ewige (im Original gesperrt).

55 F. Nietzsche: Die fröhliche Wissenschaft, 570. Erich Heller mutmasst, dass die vielen «Fragezeichen und Konjunktive, die den Stil des Absatzes bestimmen [...], den Eindruck erwecken, als wäre die ewige Wiederkunft ihrer selbst noch nicht ganz gewiß»; Erich Heller: Nietzsches Terror. Die Zeit und das Unartikulierte, in: ders.: Die Bedeutung Friedrich Nietzsches. Zehn Essays, Hamburg, Zürich 1992, 245-263, hier 257. 
Sanduhr des Daseins wird immer wieder umgedreht - und du mit ihr, Stäubchen vom Staube!» ${ }^{56}$

Im ersten Zugriff erkennt Nietzsche darin nur einen zerstörerischen Gedanken, der das Leben selbst unter einen Fluch stellt. Doch erblickt er auch eine positive Dimension, die sich exakt auf die selbstmächtige Konzeptualisierung der ewigen Lebendigkeit bezieht. Der brutale Gedanke einer ewigen Wiederkunft erweist sich zum Schluss als schärfste Kontrolle der Performierung des Willens zur Macht, denn immer hält er die Frage wach: «[W]illst du diess noch einmal und noch unzählige Male? $\gg^{57}$ Und das bedeutet: Der Übermensch als Realisierung des Machtwillens hat Wahrheitserkenntnis, Geschichte und Moral immer als eine ewige zu gestalten. ${ }^{58}$

Nietzsches Kritik an den überkommenen Wahrheitsauffassungen von Metaphysik und Moral ist gleichermassen auch eine leidenschaftliche Kritik an christlichen Wahrheitsansprüchen gewesen. In der Spätschrift Götzen-Dämmerung diskutiert er die «vier grossen Irrthümer». ${ }^{59}$ Der dritte dieser vier Irrtümer ist der «Irrthum von den imaginären Ursachen», ${ }^{60}$ und $\mathrm{zu}$ diesem Irrtum gehört nach Nietzsche auch der «gesamte Bereich der Moral und Religion» ${ }^{61}$ In seiner hochpolemischen Spätschrift Der Antichrist gilt das Christentum insgesamt als «heilige[ ] Lüge», ${ }^{62}$ als die «Kunst,

56 F. Nietzsche: Die fröhliche Wissenschaft, 570.

57 Ebd.

58 Vgl. dazu auch E. Heller: Nietzsches Terror, 259-260 sowie Tom Kleffmann: Nietzsches Begriff des Lebens und die evangelische Theologie. Eine Interpretation Nietzsches und Untersuchungen zu seiner Rezeption bei Schweitzer, Tillich und Barth, Tübingen 2003, 255-256.

59 F. Nietzsche: Götzen-Dämmerung, 88 (im Original gesperrt). Bei den vier grossen Irrtümern handelt es sich im Einzelnen um den «Irrthum der Verwechselung von Ursache und Folge» (ebd. [im Original gesperrt]), den «Irrthum einer falschen Ursächlichkeit» (ebd., 90 [im Original gesperrt]), den «Irrthum von den imaginären Ursachen» (ebd., 92 [im Original gesperrt]) und der «Irrthum vom freien Willen» (ebd., 95 [im Original gesperrt]).

$60 \quad$ Ebd., 92 (im Original gesperrt).

61 Ebd., 94 (im Original gesperrt).

62 F. Nietzsche: Der Antichrist. Fluch auf das Christentum, in: KSA VI 208. 
heilig zu lügen», ${ }^{63}$ und das, "was als Gott verehrt wurde [...] nicht nur als Irrthum, sondern als Verbrechen am Leben». ${ }^{64}$

Irrtum und Erkenntnis in der Religion

Jesus - «Ich bin [...] die Wahrheit ...»

Während in den drei synoptischen Evangelien Wahrheit beziehungsweise wahre Erkenntnis als theologisches Konzept eine untergeordnete Rolle spielt, taucht es im Johannesevangelium unübersehbar auf und ist schon als das «heimliche Thema des zweiten Teils des Evangeliums» bezeichnet worden. ${ }^{65}$ Bereits im berühmten Prolog heisst es, dass mit dem fleischgewordenen

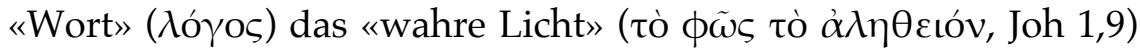
in die Welt kam und dass es «voller Gnade und Wahrheit» war

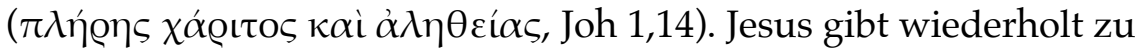
verstehen, dass sein «Zeugnis wahr» ist (z.B.: $\alpha \lambda \eta \eta \eta \dot{\zeta} \varsigma \dot{\varepsilon} \sigma \tau \iota v \dot{\eta}$ $\mu \alpha \varrho \tau v \varrho i ́ \alpha \mu$ ov, Joh 5,32). In der «Lebensbrotrede» sagt er: «[M]ein Fleisch ist wahre Speise und mein Blut ist wahrer Trank» ( $\eta \gamma \dot{\alpha} \varrho$

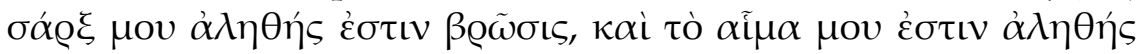

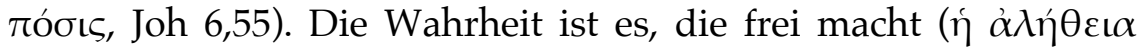
$\dot{\varepsilon} \lambda \varepsilon v \theta \varepsilon \varrho \omega \sigma \varepsilon \iota \dot{v} \mu \tilde{\alpha} \varsigma$, Joh 8,32), Jesus ist der «wahre Weinstock» $(\dot{\varepsilon} \gamma \omega$

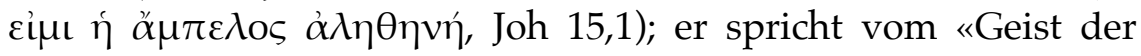

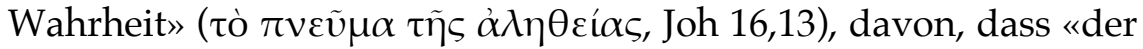

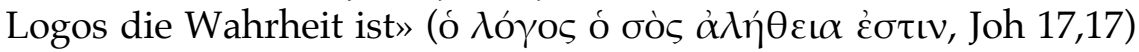
und davon, dass er «für die Wahrheit zeug[t]» ( $\mu \alpha \varrho \tau v \varrho \eta ́ \sigma \omega \tau \tilde{\eta}$ $\alpha \lambda \eta \theta \varepsilon i ́ \alpha$, Joh 18,37). Und nicht zu vergessen der «christologische

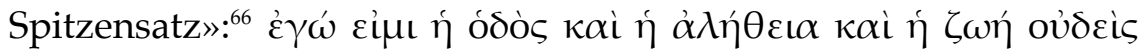

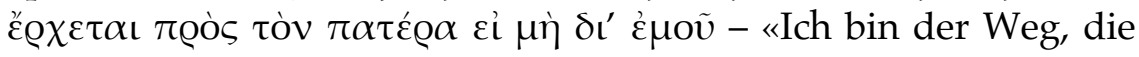
Wahrheit und das Leben, niemand kommt zum Vater, denn durch mich» (Joh 14,6).

63 Ebd., 219.

64 Ebd., 225 (im Original gesperrt).

65 Walter Klaiber: Das Johannesevangelium, II, Göttingen 2018, 5.

66 Udo Schnelle: Das Evangelium nach Johannes, Leipzig ${ }^{32004,} 253$. 
Das ist eines der berühmtesten Worte aus dem Johannesevangelium. Wahre Erkenntnis Gottes, also was Gott ist, kann nur anhand der Person Jesu erkannt werden. Nur Jesus Christus ist der Schlüssel, der Zugang zu Gott verschafft. Mehr noch: Jesus ist die Wahrheit selbst: «Jesus ist aber nicht nur der Weg zu Gott, er ist die Verkörperung göttlicher Wahrheit und göttlichen Lebens. [...] Damit formuliert Johannes einen nicht mehr zu überbietenden Exklusivitätsanspruch. ${ }^{67}$ Jesus Christus ist nicht nur Zeuge der Wahrheit, sondern die Wahrheit selbst. Die Ausschließlichkeit der Manifestation göttlicher Wahrheit in Jesus Christus richtet sich kritisch gegen konkurrierende Ansprüche. Wahrheit und Leben im umfassenden Sinne sind für Menschen nicht verfügbar, es gibt sie nur bei Jesus Christus.» ${ }^{68}$

Das Johannesevangelium statuiert indes Jesus nicht nur formal als Wahrheit; das theologische Wahrheitskonzept wird auch inhaltlich gefüllt. Die Wahrheit, für die Jesus steht, ist nichts anderes als die Wahrheit der göttlichen Liebe selbst: «Der joh[anneische] Absolutheitsgedanke ist [...] eine Variation der Absolutheit der göttlichen Liebe zu den Menschen in Jesus Christus. Der Wahr-

67 Der hier zum Ausdruck kommende Exklusivitätsanspruch wird auch in anderen biblischen Texten greifbar. Vgl. Joachim Reger: Die Absolutheit des Christentums als heilende Zumutung, Onlinepublikation LMU München 2008 (https://doi.org/10.5282/ubm/epub.3295), 2-3. So werden exklusive Momente greifbar, insofern mit dem Kommen Christi die Fülle der Zeit konkret geworden ist (vgl. Mk 1,15; Gal 4,4; Eph 1,16 u.ö.). Damit verbunden ist der Glaube, dass Jesus der einzige Mittler zwischen Gott und den Menschen ist: «Einer ist Gott, Einer auch Mittler zwischen Gott und den Menschen: der Mensch Jesus Christus» (1 Tim 2,5). Daraus folgt die universale Heilsmittlerschaft Christi: «In keinem anderen ist Heil, und es ist unter dem Himmel den Menschen kein anderer Name gegeben, worin sie heil werden können» (Apg 4,12). Dieser Heilsuniversalismus findet seinen Ausdruck in der Überzeugung von der Heilsnotwendigkeit der Taufe: «Wer glaubt und sich taufen lässt, wird gerettet; wer nicht glaubt, wird verdammt» (Mk 16,16); «Jesus antwortete: Amen, amen, ich sage dir: Wenn jemand nicht aus Wasser und Geist geboren wird, kann er nicht in das Reich Gottes kommen» (Joh 3,5).

68 U. Schnelle: Evangelium nach Johannes, 252. 
heitsanspruch Jesu ist die Unbedingtheit der Liebe». ${ }^{69}$ Diese unbedingte Liebe entspricht der göttlichen Wirklichkeit. ${ }^{70}$

Es wurde oben gesagt, dass sich die christologische Spitzenaussage Joh 14,6 kritisch gegen konkurrierende Ansprüche richtet. Das heisst, der durch Jesus Christus möglichen wahren Gotteserkenntnis stehen konkurrierende Ansprüche gegenüber. Was sind das für Ansprüche? In Joh 18,36 äussert Jesus im Gespräch mit Pilatus den bekannten Satz: «Mein Reich ist nicht von dieser Welt» das fragliche Gespräch endet im Übrigen mit der berühmten Frage des Pilatus «Was ist Wahrheit?» (Joh 18,38). ${ }^{71}$ Daraus lässt sich direkt ableiten, dass es sich bei den im Raum stehenden konkurrierenden Ansprüchen um Ansprüche der Welt handelt.

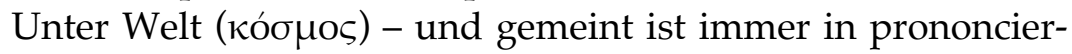
tem Sinne diese Welt (ó kóouos oũ̃os) - versteht das Johannesevangelium in erster Linie die Menschenwelt. ${ }^{72}$ Charakterisiert ist diese Welt vor allem durch vier Momente: Finsternis, Knechtschaft, Tod und - für unseren Zusammenhang am wichtigsten - Un-

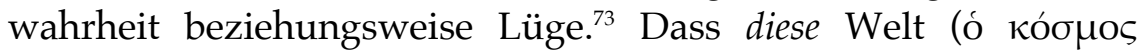
oũtos) wesenhaft eben auch Unwahrheit und Lüge ist, wird einerseits implizit deutlich, und zwar dadurch, dass es die Aufgabe des inkarnierten Logos ist, von der Wahrheit zu zeugen. Vor allem im 8. Kapitel des Johannesevangeliums wird das Wesen des Kosmos als Lüge auch explizit benannt. Jesus hält den Juden vor, dass sie ihm nicht glauben, weil sie vom Teufel abstammen. Und der Teufel wird expressis verbis als Lügner und Vater der Lüge bezeichnet

Ebd.

Vgl. dazu Rudolf Bultmann: Theologie des Neuen Testaments, Berlin (Ost) 1970, 371-373: «Vielmehr ist die Grundbedeutung von $\alpha \lambda \eta \dot{\theta} \theta \varepsilon \iota \alpha$ bei Johannes die der Wirklichkeit Gottes, die, da Gott der Schöpfer ist, die einzige, echte Wirklichkeit ist. [...] Wenn Gottes Wort die Wahrheit ist (Joh 17,17), so deshalb, weil in ihm Gottes Wirklichkeit offenbar wird.»

1 Bultmann interpretiert die Frage des Pilatus als «abweisende Frage», die zum Ausdruck bringt, «[d]aß der Welt die Wirklichkeit Gottes gleichgültig geworden ist», ebd., 371 .

Vgl. ebd., 367.

Vgl. ebd., 368. 


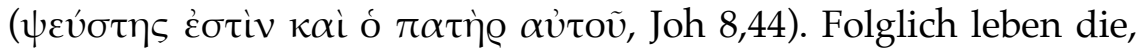
die nicht an Jesus glauben, in der Sphäre der Lüge, der Unwahrheit. Der theologischen Logik des Johannesevangeliums entsprechend ist es die durch Jesus verkörperte Wahrheit, die aus der Welt der Unwahrheit und Lüge befreit: «Die Wahrheit macht euch frei» - $\dot{\eta} \dot{\alpha} \lambda \dot{\eta} \theta \varepsilon\llcorner\alpha \dot{\varepsilon} \lambda \varepsilon v \theta \varepsilon \varrho \omega ́ \omega \varepsilon\llcorner\dot{v} \mu \tilde{\alpha} \varsigma$ (Joh 8,32).

Es gehört zu den grossen Irritationen der Kirchengeschichte, dass das fragliche Spitzenzitat («Ich bin der Weg, die Wahrheit etc.») zum Ausgangspunkt zum Teil verheerender Irrtümer geworden ist. Denn vor allem mithilfe dieses Ausspruches wurde die Absolutheit des Christentums begründet, also diejenige Vorstellung, dass das Christentum und nur das Christentum die höchste und vollendete Religion sei. ${ }^{74}$ Der Absolutheitsanspruch figurierte dann als Begründung für äusserst fatale theologische Entscheidungen und für entsprechendes kirchliches Handeln.

Die Vorstellung von der Absolutheit des Christentums resultierte zunächst daraus, dass die älteste Christenheit inmitten einer Unzahl von Kulten und Religionen situiert war, die zu grossen Teilen entweder exklusive Mysterienkulte und Arkanreligionen oder Nationalreligion und sogenannte religio licita (also eine «erlaubte Religion») waren. Von diesen Religionen und Kulten grenzte sich das junge Christentum ab, setzte sich absolut, ganz im Sinne der ursprünglichen Bedeutung von absolutus im Sinne von «für sich bestehend [...] von nichts anderem abhängig». ${ }^{75}$ Absolutheit meint also zunächst ganz allgemein ein Losgelöstsein von vorgegebenen religiösen und kulturellen Strukturen. ${ }^{76}$

Diese zunächst negative Absolutheit wird im 4. Jahrhundert zu einer positiv-dogmatischen Absolutheit. Sie findet ihren Ausdruck einerseits in den Festsetzungen des Konzils von Nicäa (325),

74 Zur Absolutheit des Christentums ausführlich: Reinhold Bernhardt: Der Absolutheitsanspruch des Christentums. Von der Aufklärung bis zur Pluralistischen Religionstheorie, Gütersloh 1990.

75 Heinrich Georges: Ausführliches lateinisch-deutsches Handwörterbuch, I, Gotha 1913, 34 (im Original gesperrt).

76 Vgl. Hans-Georg Fritzsche: Lehrbuch der Dogmatik, I, Berlin (Ost) 1964, 210. 


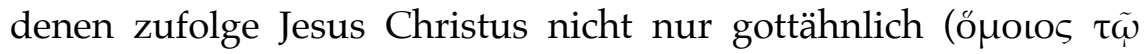

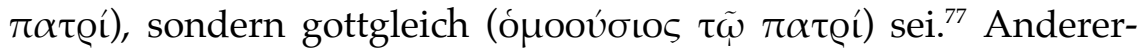
seits kulminiert sie in der auf Cyprian zurückgehenden Formel «extra ecclesiam nulla salus». Es ist dann exakt diese dogmatisch verfestigte Absolutheit des Christentums gewesen, die zu den zahlreichen Irrtümern und Verirrungen der Kirchengeschichte geführt hat: Kreuzzüge, Zwangschristianisierungen, imperialistisches Gebaren der Päpste, Inquisition und Hexenverfolgung seien hier nur als die unrühmlichsten Verirrungen aufgerufen.

Der Absolutheitsanspruch des Christentums ist in der Neuzeit mehr und mehr erodiert. Die Gründe dafür waren auf der einen Seite die Wahrnehmung einer inneren Entwicklung des Christentums selbst. Das wirft die Frage auf: Wie kann die Absolutheit des Christentums mit der Tatsache in Verbindung gebracht werden, dass dieses sich entwickelt hat und scheinbar kaum noch in Kongruenz mit seinen Ursprüngen zu bringen ist? ${ }^{78}$ Auf der anderen Seite wird die Absolutheit des Christentums relativiert durch die Begegnung vor allem mit fernöstlichen Religionen und durch die sukzessive Einsicht, dass auch diese Religionen «Wahrheitsmomente und positive Funktionen ${ }^{79}$ enthalten.

Ihren exemplarischen Ausdruck findet die neuzeitliche Erosion des christlichen Absolutheitsanspruchs in Lessings berühmter Ringparabel, der zufolge das, was allen Religionen gemeinsam ist, die wahre oder natürliche Religion sei: «herzliche[ ] Verträglichkeit, [...] Wohltun, [...] innigste[ ] Ergebenheit in Gott». ${ }^{80}$ Alle positiven Religionen, sofern sie Realisierung des natürlichen Kerns sind, gelten folglich gleich viel oder gleich wenig.

Der hieraus folgende radikale Relativismus - es ist dann im Grunde gleich, welcher Religion man angehört - hat freilich Widerspruch evoziert. Eine der originellsten und wirkmächtigsten

77 Vgl. ebd., 210-212.

78 Vgl. J. Reger: Die Absolutheit des Christentums, 4.

79 Vgl. H.-G. Fritzsche: Lehrbuch der Dogmatik, 211.

80 Gotthold Ephraim Lessing: Nathan der Weise, in: Werke. In sechs Bänden, II, hg. von Ernst Consentius, Berlin o.J., 337. 
Antworten auf diesen Relativismus und die Bedeutung des Absolutheitsparadigmas hat im 19. Jahrhundert Albrecht Ritschl vorgelegt. Ritschl hält an der Absolutheit des Christentums fest und relativiert sie gleichzeitig, indem er ihr eine nur regulative Funktion zuschreibt. ${ }^{81}$

Grundsätzlich gibt es für Ritschl nicht die allgemeine, natürliche Religion, sondern nur konkrete, historisch-vorfindliche, sprich positive Religionen. Ein Allgemeinbegriff der Religion kann demzufolge nur aus dem religionswissenschaftlichen Vergleich der verschiedenen Religionen hervorgehen. Es ist «die Beobachtung und Vergleichung der einzelnen geschichtlichen Religionen, aus welchen der allgemeine Begriff abstrahiert wird». ${ }^{82} \mathrm{Da}$ aber in den Augen Ritschls das Christentum die höchste, absolute und sonach einzig wahre Religion repräsentiert, kann - wie auch das von ihm eingeschlagene Verfahren belegt - der allgemeine Begriff der Religion nur anhand des Christentums gebildet werden. Ansonsten kämen religiöse Gebilde mit in Betracht, die den Sachverhalt Religion im Vollsinn gar nicht erfüllen würden. Das wiederum bedeutet, dass der aufgestellte allgemeine Religionsbegriff erklärt christliche Züge trägt, und er demzufolge, wie Ritschl festhält, «nicht unbestimmt genug» ${ }^{83}$ ist. Dieser ungenügenden Unbestimmtheit möchte Ritschl mit der angedeuteten Restriktion der Reichweite des allgemeinen Religionsbegriffs begegnen. ${ }^{84}$

Zum Folgenden vgl. auch Matthias Neugebauer: Lotze und Ritschl. Reich-Gottes-Theologie zwischen nachidealistischer Theologie und neuzeitlichem Positivismus, Frankfurt a.M. u.a. 2002, 103-106.

22 Albrecht Ritschl: Rechtfertigung und Versöhnung. Die positive Entwicklung der Lehre, Bonn ${ }^{3} 1888,187$.

Ebd., 185.

Es ist klar, dass - wie oben angemerkt - die vorausgesetzte Absolutheit des Christentums trotz der doppelten Spannung das eigentliche Grundproblem darstellt. Denn würde Ritschl nicht von der theoretischen Höchstgeltung des Christentums überzeugt sein, so käme von vornherein die partikulare, historische Religion des Christentums nicht als Kandidat zur Erhebung des Allgemeinbegriffs in Frage. 
Ritschl notiert: «Der Allgemeinbegriff der Religion findet in der Erforschung des Christenthums regulativen Gebrauch». ${ }^{85}$ Dies bedeutet zweierlei: Zunächst - was den methodischen Status der Religionstheorie betrifft - hat der aufgestellte Allgemeinbegriff der Religionstheorie «nicht den Anspruch, eigentliche Definition des Gattungsbegriffs von Religion zu sein». ${ }^{86}$ Das heisst, der am Christentum als der absoluten Religion entworfene Religionsbegriff prätendiert nicht mehr eine Anwendbarkeit auf alle geschichtlich hervorgetretenen Religionen. Insofern er aus erklärt christlicher Perspektive entworfen ist, hat er seine Berechtigung auch nur weitgehend in den Bezugsebenen des Christentums. Insofern hat er nur noch binnenchristliche Geltungskraft. Eine Hierarchisierung der verschiedenen Religionen, an deren erster Stelle das Christentum steht, kann Ritschl vor dem Hintergrund der eingeschränkten Reichweite des Religionsbegriffs nicht mehr objektiv festschreiben. Er verzichtet auf ein solches Prozedere als «ziellos und unausführbar». ${ }^{87}$

Wenn Ritschl dennoch das Christentum als «die höchste unter allen Religionen und die vollkommenste ${ }^{88}$ bezeichnet, so hat dies nur binnenchristliche Relevanz und verweist auf den angekündigten zweiten Punkt, der die spezifische Funktion und Leistung des Religionsbegriffs innerhalb des Christentums betrifft. ${ }^{89}$ Der Religionsbegriff hat in seiner begrenzten Reichweite «die wissenschaftliche Aufgabe zur Verständigung der Christen untereinander».90 Diese Funktion erfüllt sich, indem das Verhältnis zu den anderen Religionen geklärt wird. Der allgemeine Religionsbegriff wird nur

85 A. Ritschl: Rechtfertigung und Versöhnung, 187 (im Original zum Teil gesperrt, Hervorhebung M.N.).

86 Ebd., 185.

87 Ebd., 188.

88 Ebd.

89 Zum Folgenden vgl. besonders Arnulf von Scheliha: Der Glaube an die göttliche Vorsehung. Eine religionssoziologische, geschichtsphilosophische und theologiegeschichtliche Studie, Stuttgart, Berlin, Köln 1998, 244-245.

$90 \quad$ A. Ritschl: Rechtfertigung und Versöhnung, 188. 
noch als interner $«$ Normbegriff ${ }^{91}$ aufgefasst, der aus der Perspektive des Christentums angibt, zu welchem Grad an religiöser Suffizienz Religionen «als Glieder der geistigen Geschichte der Menschheit $\gg^{92}$ prinzipiell fähig sind. Die anderen Religionen erscheinen vom Standpunkt des Christentums zwar als «Modificationen ins Unvollkommene», ${ }^{93}$ aber dies dient gerade nicht der Konfirmierung einer objektiven Stufenfolge der Religionen, sondern wesentlich der internen oder subjektiven Vergewisserung der ureigensten christlichen Position. ${ }^{94}$

Um es genau zu sagen: Es geht mitnichten um eine Rettung der Absolutheit des Christentums. Das ist nicht gewollt, und das ist auch ein falscher apologetischer Instinkt. Nein, es geht um die gewissenhafte Restringierung und Realisierung von individuellen und intersubjektiven Glaubenspositionen. Und das bedeutet nichts anderes, als dass Ritschl eine bis heute unüberholte Sortierung der Problemvariablen hergestellt hat. Diese wird vielfach verkannt und meint dabei dennoch etwas zutiefst Einleuchtendes: Wer nicht mit der Evidenz seines Glaubenssystems und seiner religiösen Überzeugungen rechnen darf, der wird sich in Synkretismus, ungeklärtem Atheismus, nicht reflektiertem Agnostizismus oder in weltanschaulicher Beliebigkeit verlieren.

Denn: Es gehört zu den theologischen und religionssoziologischen Merkmalen von Religionszugehörigkeit, dass das Überzeugtsein von der Richtigkeit des eigenen religiösen Erklärungssystems zu den Grundtatsachen einer religiösen Einstellung gehört. Wäre ich nicht von der Evidenz meines religiösen Glaubens überzeugt, wäre ich kein ernstzunehmender Teilnehmer, keine ernstzunehmende Teilnehmerin des fraglichen Systems, und es würden auch nicht die fraglichen religiösen Instinkte bedient werden. Die in

91 Vgl. Rolf Schäfer: Ritschl. Grundlinien eines fast verschollenen dogmatischen Systems, Tübingen 1968, 165.

92 A. Ritschl: Rechtfertigung und Versöhnung, 187.

93 Ebd., 188.

94 Vgl. Notgar Slenczka: Der Glaube und sein Grund. F. H. R. von Frank und seine Auseinandersetzung mit A. Ritschl und die Fortführung seines Programms durch L. Ihmels, Göttingen 1998, 245. 
Frage stehende Restriktion meint nicht mehr, aber auch nicht weniger als religiöse Selbstbestimmung, -verortung und -behauptung unter den Bedingungen des Pluralismus.

Ritschls Position könnte man heute auch als Filterblaseneinstellung beschreiben. Das ist phänomenologisch betrachtet auch gar nicht so unzutreffend. Denn Religion kann beispielsweise die Vision einer heilen Welt inmitten von globalen Verwerfungen aufrecht halten. Aber als Blase ist es eine dynamische Blase. Sie kann, will und muss kontrafaktisch-faktisch sein und die Welt des Augenscheins und die Welt der vermeintlichen Selbstverständlichkeiten produktiv hinterfragen. Genau das ist der Sinn reformatorischen Christseins.

\section{Schluss}

Anhand des Politikers Donald Trump, des Philosophen Friedrich Nietzsche und des johanneischen Jesus wurden drei Facetten des Verhältnisses von Irrtum und Erkenntnis entfaltet. Diese lassen sich unschwer als die systematischen Positionen von Irrtum ohne Erkenntnis, Erkenntnis als Irrtum und Erkenntnis und Irrtum erkennen. Zum Schluss soll ein Blick auf das Verhältnis dieser Positionen $\mathrm{zu}$ - und untereinander geworfen werden. Und dabei ist es so, dass sich diese Positionen wechselseitig kritisieren und korrigieren.

Erstens: Ein Politikstil, wie ihn Trump verkörpert und der «America first» und die Unfehlbarkeit des Staatslenkers bewirtschaftet, weiss sich durch Nietzsche herausgefordert einerseits durch den Anspruch der Wahrhaftigkeit und dadurch, Entscheide gewissermassen sub specie aeternitatis zu fällen - eine in der Tat übermenschliche Aufgabe. Aber auch von Seiten der Religion und Theologie kommen Herausforderungen. Es war kein geringerer als Paul Tillich, der ein derartiges Staats- und Regierungsverständnis mit dem Begriff des Dämonischen in Verbindung gebracht hat. Dämonisch bedeutet bei Tillich vor dem Hintergrund seiner Unterscheidung von Form und Gehalt, dass die Formen sich verabso- 
lutieren und die Grundlagen der Gemeinschaft von axiologischen Fundamenten praktisch frei sind. ${ }^{95}$ Das sind profane Dämonien. Sie tragen die Signatur der «Hybris des Rationalen». ${ }^{96}$ Und aus ihnen folgt nichts anderes als Entleerung: Entleerung vor allem in moralischer, ästhetischer und religiöser Hinsicht. ${ }^{97}$

Zweitens: Nietzsches These von der Irrtümlichkeit der Wahrheit muss sich einerseits die Frage gefallen lassen, welchen Wahrheitsanspruch diese These für sich selbst beansprucht und was bleibt, wenn die wahre und die scheinbare Welt abgeschafft würden. Mit der Einsicht in den Metapherncharakter jeder Wahrheitsbehauptung können wir den «Trieb zur Wahrheit, zum reinen Erkennen ${ }^{98}$ nicht einfach ignorieren, aber auch nicht ohne Weiteres von Irrtum, Perspektivität und Schein lösen. ${ }^{99}$ Hinzu kommt, dass es keineswegs ausgemacht ist, dass das begrifflich-diskursive Denken ohne weiteres hintergehbar ist. ${ }^{100}$ Und schliesslich stellt

95 Paul Tillich: Grundlinien des religiösen Sozialismus, in: Gesammelte Werke, II, Stuttgart 1962, 91-119, hier 98: «Das Dämonische ist die Erhebung des irrationalen Grundes aller individuellen schöpferischen Formverwirklichung im Widerspruch mit der unbedingten Form. [...] Das Dämonische ist anschaubar wie das Göttliche in dem Ekstatischen, Übermächtigen, Schauervollen. Während aber die Ekstatik des Göttlichen die unbedingte Form bejaht und darum Formen schafft, zerstört die Ekstatik des Dämonischen die Form.»

96 Ebd., 103. Vgl. dazu Matthias Neugebauer: Die Ethik-Konzeption Paul Tillichs. Eine Annäherung mit Rücksicht auf das Gesamtwerk, in: Christian Danz, Marc Dumas, Werner Schüßler, Mary Ann Stegner, Erdmann Sturm (Hg.): Internationales Jahrbuch für die Tillich-Forschung, X, Berlin, Boston 2015, 103-141, hier 119-122.

97 Ebd.: «Sobald [...] [der] Gehalt aber schwindet, bleibt nichts übrig als die leere Erhabenheit der autonomen Form.»

98 F. Nietzsche: Ueber Wahrheit und Lüge, 882.

99 Vgl. Michael Pfister: Über Friedrich Nietzsche: Über Wahrheit und Lüge im aussermoralischen Sinne; Die fröhliche Wissenschaft; Zur Genealogie der Moral, in: Theorie Kritik, 6.11.2016, http:/ / www.theoriekritik.ch/?p=2988 (30.1.2019).

100 Vgl. Volker Gerhardt: Friedrich Nietzsche, München ${ }^{3} 1999$, 65: «Die Philosophie kann auf das Begreifen nicht verzichten; und da jeder Begriff ursprünglich auf etwas Allgemeines zielt, liegt in ihm selbst schon von selbst ein Vorbegriff auf einen prinzipiell erkennbaren Zusammenhang. 
sich die Frage, ob Nietzsche bei aller Kritik an Metaphysik, Moral und Religion nicht selbst wieder metaphysische Paradigmata, moralische Imperative und unter der Hand eine neue Religion etabliert, dessen Prophet Zarathustra ist. ${ }^{101}$ Und nur am Rande sei hier noch einmal erwähnt, dass die Wiederkunftslehre von einem nächtlichen Dämon eingeführt wird, womit wieder die Thematik des Dämonischen aufgerufen ist.

Drittens: Auch Religion, auch der christliche Glaube und die christliche Theologie dürfen und müssen sich kritisch befragen lassen. Es ist das Rettende und zugleich das Gefährliche, dass Religionen mit göttlichen Wahrheiten handeln, sie kompetent verwalten, segensreich weitergeben - aber eben auch zu politischen Zwecken und zur Demagogie missbrauchen können. Ein Christentum, das mit den steilen Ansprüchen eines johanneischen Christus unreflektiert operiert, gerät notorisch in Gefahr, den beschriebenen Irrtümern anheim zu fallen. Mehr noch und um abschliessend noch einmal auf Tillich zurückzukommen: Tillich kennt nicht nur die beschriebenen Gestalten sogenannter naturaler Dämonien, sondern auch sakrale Dämonien. Bei ihnen handelt es sich genau um die Umkehrung, dass also ein religiöser Gehalt alle Formen vollständig okkupiert. Sie sind in negativer Hinsicht gekennzeichnet durch «Niederhaltung und Zerstörung der geistigen Freiheit und Schöpferkraft». ${ }^{102}$ Ein solches dämonisches Christentum hat in der Moderne freilich keinen Platz mehr. Und mit Blick auf Nietzsche endlich müssen sich auch Religion und Theologie auf ihren Umgang mit Symbolen

Man denkt immer schon systematisch, sofern man nur denkt, auch wenn man die abschließenden Systeme mit guten Gründen verwirft. Mit jedem Begriff ist die bloß sinnliche Gegenwart überschritten und der Keim zum System gelegt.»

101 Es sei dahingestellt, ob Zarathustra Nietzsches (einziges) alter ego ist. Volker Gerhardt hat die These stark gemacht, dass eine der Masken, hinter denen Nietzsche sich versteckt, auch Sokrates sein könnte. Vgl. Volker Gerhardt: Nietzsches Alter-Ego. Über die Rückkehr des Sokrates, in: Nietzscheforschung 8 (2001) 315-332.

102 P. Tillich: Grundlinien des religiösen Sozialismus, 102. 
und Metaphern befragen lassen. ${ }^{103}$ Das steht jedoch auf einem anderen Blatt.

Zum Schluss ist es mit Blick auf Irrtum und Erkenntnis wohl so, dass niemand und auch keine Disziplin die Wahrheit gepachtet hat: weder Politik, noch Philosophie und auch keine Religion, sondern dass in der Postmoderne immer von einem offenen und hybriden Wahrheitsverständnis auszugehen ist, demnach Wahrheit immer offene Wahrheit ist. ${ }^{104}$ Und wenn das ernst genommen und beherzigt wird, dann gewinnt auch ein altes, aber durch und durch aufgeklärtes Lichtenberg-Wort wieder an Bedeutung und Aktualität: «Die Entscheidung über Irrthum und Wahrheit muß nie, nie (sic!) das Monopol eines Charakters werden, so wenig als eines Standes. Wahrheitsmonopole einem einzelnen Stande oder Charakter zu verleihen, sind Beeinträchtigungen für alle übrigen und wahre Injurien für die Menschheit». ${ }^{105}$

103 «Weitgehend akzeptiert ist, dass die religiöse Sprache entscheidend durch Metaphern und symbolische Ausdrucksweisen geprägt ist»; Klaus Tanner: Vom Mysterium des Menschen. Ethische Urteilsbildung im Schnittfeld von Biologie, Rechtswissenschaft und Theologie, in: Reiner Anselm; Ulrich H. J. Körtner (Hg.): Streitfall Biomedizin, Göttingen 2003, 135--158, hier 149.

104 Vgl. Ulrich Barth: Semantischer Interpretationismus. Die andere Selbstkorrektur der Analytischen Philosophie, in: ders.: Religion in der Moderne, Tübingen 2003, 202-232, hier 218: «Hermeneutische Wahrheit ist immer offene Wahrheit.»

105 Georg Christoph Lichtenberg: Lieutenant Greatraks, in: Vermischte Schriften, V, Göttingen 1844, 297-306, hier 305 (im Original zum Teil gesperrt). 
174 Matthias Neugebauer: Wahrheit, Irrtum und Lüge

conexus 2 (2019) 145-174

(C) 2019 Matthias Neugebauer. Dieser Beitrag darf im Rahmen der Lizenz CC BY-NC-ND 4.0 - Creative Commons: Namensnennung/nicht kommerziell / keine Bearbeitungen - weiterverbreitet werden.

\section{(c) (1) (2) \\ https:// doi.org/10.24445/ conexus.2019.02.010}

Prof. Dr. Matthias Neugebauer, Universität Zürich, Theologische Fakultät, Kirchgasse 9, 8001 Zürich matthias.neugebauer@access.uzh.ch 